\title{
Temas de investigación relevantes en ciencias administrativas: una mirada desde el análisis de redes
}

\author{
Abel Aníbal del Río-Cortina ${ }^{1}$ \\ Universidad Nacional Abierta y a Distancia - UNAD \\ abel.delrio@unad.edu.co \\ Jorge Armando Amador-Moncada² \\ Universidad Nacional Abierta y a Distancia - UNAD \\ jorge.amador@unad.edu.co \\ Rafael Ricardo Rentería-Ramos ${ }^{3}$ \\ Universidad Nacional Abierta y a Distancia - UNAD \\ rafael.renteria@unad.edu.co \\ Iván Darío Urrea-Ospina ${ }^{4}$ \\ Centro de Estudios Técnicos y Artísticos - CE-ART \\ ivanurrea@outlook.com
}

DOI: https://doi.org/10.21158/01208160.n91.2021.3093

Fecha de recepción: 24 de octubre de 2019

Fecha de aprobación: 21 de octubre de 2021

Fecha de publicación: 16 de diciembre de 2021

Cómo citar este artículo: del Río-Cortina, A. A.; Amador-Moncada, J. A.; Rentería-Ramos, R. R.; Urrea-Ospina, I. D. (2021). Temas de investigación relevantes en ciencias administrativas: una mirada desde el análisis de redes. Revista Escuela de Administración de Negocios, (91), páginas. DOI: https://doi.org/10.21158/01208160.n91.2021.3093

\section{Resumen}

Existen fuertes críticas a la baja relación que existe entre los procesos de investigación y el contexto empresarial en las facultades, los programas de administración y las escuelas de negocios poco posicionados. Este documento tiene como objetivo determinar los temas relevantes en ciencias administrativas mediante la aplicación de análisis de redes para identificar las instituciones que manejan temas recurrentes, aquellas que abordan temas especializados y los territorios con investigación en el campo administrativo. Se identificaron las mejores facultades, programas de administración y escuelas de negocios a nivel mundial y nacional y, a partir de esta selección, se construyó un modelo relacional de las interacciones entre las instituciones y las temáticas utilizando la teoría de redes. Se implementó este tipo de análisis dado que permite la revisión sistemática y sus resultados se convierten en insumos que contribuyen a la posterior toma de decisiones por parte de las instituciones inmersas en este campo. Entre los resultados se encuentran como nodos institucionales notables la Escuela de Negocios de Columbia, la Escuela de Negocios Insead, la Universidad de Negocios Cornell, la Escuela de Negocios de Harvard y, finalmente, la Escuela de Gestión SLOAN del Massachusetts Institute of Technology. Finalmente, se identificaron como nodos temáticos relevantes aquellos relacionados con finanzas, emprendimiento, globalización y liderazgo.

Palabras clave: análisis de redes; investigación en administración; ciencias administrativas; teoría de redes; nodos institucionales; nodos temáticos.

\footnotetext{
${ }^{1}$ Administrador de Empresas - Universidad de Cartagena. Magister en Desarrollo Empresarial - Universidad del Magdalena. Doctor en Gerencia de Proyectos - Universidad Ean. ORCID: https://orcid.org/0000-0002-2713-432X

${ }^{2}$ Ingeniero Químico, Magister en Ingeniería, Automatización Industrial, Doctor en Ingeniería línea Automática - Universidad Nacional de Colombia sede Manizales. ORCID: https://orcid.org/0000-0002-4296-6271

${ }^{3}$ Ingeniero Industrial - Universidad Los Libertadores. Doctor en Ciencias Económicas -Universidad de La Habana. ORCID: https://orcid.org/00000002-5857-9153

${ }^{4}$ Magíster en Educación Superior - Universidad Arturo Prat de Chile. Vicerrector académico Centro de Estudios Técnicos y Artísticos - CE-ART. ORCID: https://orcid.org/0000-0003-1026-2402
} 


\title{
Relevant Research Topics in Administrative Sciences: a View from the Analysis of Networks
}

\begin{abstract}
There is a great deal of criticism of the low relationship between the research processes and the business context in faculties, the management programs, and the poorly positioned business schools. This paper aims to determine relevant topics in administrative sciences by applying the analysis of networks to identify the institutions that handle recurrent topics, those that address specialized topics, and the territories that include research in the administrative field. The study identified the best worldwide and nationwide faculties, management programs, and business schools. We built a relational model of the interactions between institutions and topics using the network theory from this selection. This type of analysis was implemented since it allows a systematic review, and the results become inputs that contribute to subsequent decision-making by institutions involved in this field. We identified the Columbia Business School, INSEAD Business School, Cornell Business College, Harvard Business School, and, finally, the SLOAN School of Management of the Massachusetts Institute of Technology as notable institutional nodes. Finally, the relevant thematic nodes were identified: related to finance, entrepreneurship, globalization, and leadership.
\end{abstract}

Keywords: network analysis; management research; management science; network theory; institutional nodes; thematic nodes.

\section{Temas de pesquisa relevantes em ciências administrativas: um olhar a partir da análise de redes}

\section{Resumo}

Há fortes críticas à pouca relação que existe entre os processos de pesquisa e o contexto empresarial em faculdades, programas de administração e escolas de negócios mal posicionadas. 0 objetivo deste documento é determinar os temas relevantes nas ciências administrativas por meio da aplicação da análise de redes para identificar as instituições que tratam de temas recorrentes, as que tratam de temas especializados e os territórios com pesquisas na área administrativa. Foram identificadas as melhores faculdades, programas de administração e escolas de negócios em nível mundial e nacional e, a partir dessa seleção, foi construído um modelo relacional das interações entre instituições e os temas utilizando a teoria das redes. Esse tipo de análise foi implementado por permitir uma revisão sistemática e por seus resultados se tornarem insumos que contribuem para a posterior tomada de decisão por parte das instituições imersas nesta área. Entre os resultados foram encontrados como nós institucionais notáveis a Escola de Negócios de Columbia, a Escola de Negócios Insead, a Universidade de Negócios Cornell, a Escola de Negócios de Harvard e, finalmente, a Escola de Gestão SLOAN do Instituto de Tecnologia de Massachusetts. Finalmente, foram identificados como nós temáticos relevantes, aqueles relacionados a finanças, empreendedorismo, globalização e liderança.

Palavras-chave: análise de redes; pesquisa em administração; ciências administrativas; teoria de redes; nós institucionais; nós temáticos. 
L'analyse des réseaux :

une thématique pertinente de recherche en gestion

\begin{abstract}
Résumé
De fortes critiques existent au sujet du manque relations existantes entre les processus de recherche et d'investigation et l'environnement entrepreneurial et des affaires dans les facultés et programmes de gestion des écoles de commerce de second rang. Cet article tentera de déterminer les thématiques pertinentes de recherche en gestion au travers de l'analyse des réseaux pour identifier les institutions traitant des thèmes récurrents, se positionnant sur des thématiques spécialisées, et les champs d'investigation dans le domaine du commerce et de l'entrepreneuriat. L'identification des meilleures universités, programmes de gestion et écoles de commerce a été réalisée au niveau mondial et, à partir de cette sélection, nous avons construit un modèle relationnel des interactions entre institutions et thèmes abordés sur la base de la théorie des réseaux. Ce type d'analyse permet une révision systématique des résultats qui deviennent des intrants pour la prises de décision ultérieures des institutions spécialisées du secteur. Les réseaux institutionnels les plus efficients sont le fait de la Columbia Business School, l'Insead Business School, Cornell Business University, Harvard Business School ou la SLOAN - School of Management du Massachusetts Institute of Technology. Enfin, les réseaux et thématiques pertinentes semblent être liés à la finance, à l'entrepreneuriat, à la mondialisation et au leadership.
\end{abstract}

Mots-clés: analyse de réseau; recherche en gestion; sciences de gestion; théorie des réseaux; réseaux institutionnels; réseaux thématiques.

\title{
1. Introducción
}

La gestión del conocimiento es un factor que sirve de apoyo para la transformación local, regional, nacional e internacional, en la medida en que se encuentre en contacto permanente con el contexto territorial (Dalkir, 2005). De manera consecuente, en el presente documento se consideran los temas de investigación abordados por las mejores facultades, programas de administración y escuelas de negocios tanto mundiales como nacionales, con el objetivo de determinar los temas relevantes en ciencias administrativas, lo cual sirve de base para la adopción de los mejores referentes dentro de un proceso de identificación de actores comparables para un posterior benchmarking (Nazarko, Kuzmic, Szubzda-Prutis y Urban, 2009).

La identificación de los mejores referentes es el soporte de una toma de decisiones que lleve a las instituciones de educación superior que manejan temas de investigación en ciencias administrativas a desarrollar isomorfismo competitivo (Meyer, 1979; Fenney, 1980), en 
contraposición con el isomorfismo institucional (Dimaggio y Powell, 1983), es decir, un proceso en el que se aborden los temas que correspondan con la visión y las características institucionales que más se aproximen al contexto propio, dentro de parámetros de adopción de buenas prácticas de diferenciación institucional.

Enfrentar los desafíos de los procesos de investigación en ciencias administrativas requiere del fortalecimiento de alianzas y de redes de cooperación, en el marco de la generación de espacios de aprendizaje y de análisis de temas de investigación pertinentes en el contexto local y nacional, pero que, a su vez, guarden una relación significativa con las problemáticas globales, siendo temas académicos relevantes que impactan tanto el sector productivo como la comunidad académica.

La gestión de los procesos de investigación en las facultades, en los programas de administración y en las escuelas de negocios presenta la complejidad inherente a los desafíos de las instituciones de educación superior en el contexto de la internacionalización (Guillotin y Mangematin, 2015). De esta manera, precisa de mecanismos que, enmarcados en modelos de gestión estratégica (David, 2013), sean un soporte para la generación de mejores intervenciones que fortalezcan el desarrollo curricular y propicien la generación de capacidades institucionales (Danielson, Doolittle y Bradley, 2007).

Las facultades, los programas de administración y las escuelas de negocios poco posicionados han sido objeto de críticas en el ámbito internacional, debido a que presentan procesos de investigación alejados de las necesidades del sector productivo y del contexto real, lo cual se evidencia en lo planteado en el 2012 por The Association to Advance Collegiate Schools of Business (AACSB):

El rápido cambio en el tamaño y la estatura de la investigación en las escuelas de negocios ha generado un diálogo y un debate apasionados. Por ejemplo, las escuelas de negocios han sido criticadas recientemente por poner demasiado énfasis en la investigación en relación con la enseñanza y por producir investigaciones demasiado limitadas, irrelevantes y poco prácticas. (AACSB, 2012, p.10) 
El anterior es un escenario que, aun presentando un mejoramiento importante, supone una necesidad de mayor fortalecimiento institucional en el nivel regional, dentro de un contexto global (AACSB, 2012; Mihaela, Amalia y Bogdan, 2015; Salcedo-Serna, Londoño-Cardozo y Hernández-Madroñero, 2017), de modo que requiere del seguimiento institucional en función de estándares de excelencia (AACSB, 2020).

Lo anterior implica el análisis permanente de los parámetros del Consejo Nacional de Acreditación (2013) y la revisión de los lineamientos de organismos acreditadores internacionales como, por ejemplo, la Association to Advance Collegiate Schools of Business (AACSB), la Association of MBAs (AMBA), el European Quality Improvement System (EQUIS), y la Accreditation Council for Business Schools and Programs (ACBSP), de los que se considera la AACSB como el de mayor representatividad por su enfoque investigativo, rigurosidad y tradición (Guillotin y Mangematin, 2015). Se complementa este proceso con el análisis de ránquines internacionales que muestra el posicionamiento de las facultades, los programas de administración y las escuelas de negocios, entre los cuales el ranquin de Shanghái es el más representativo.

Con el fin de determinar los temas relevantes en ciencias administrativas se identificaron las instituciones que registran temas recurrentes, las que desarrollan temas especializados y las regiones que presentan investigación en el campo administrativo.

El análisis de redes sociales se despliega como una metodología que permite visualizar interconexiones entre las diferentes instituciones analizadas y los temas del campo administrativo abordados por dichas instituciones, en correspondencia con la complejidad de la educación superior en Colombia y, a su vez, con las necesidades de las facultades, los programas de administración y las escuelas de negocios, de manera que muestra rutas de fortalecimiento estratégico en investigación (Del Río-Cortina, 2020). 
El análisis apropiado de los temas de investigación enfocado desde una visión institucional, de facultad, programa y escuela permite generar la transición de un horizonte en el que todos los actores del sistema de investigación se comportan de forma idéntica, sin tener en cuenta factores diferenciadores en lo que se denomina «isomorfismo institucional» (Dimaggio y Powell, 1983), hacia un comportamiento estratégico producto de un análisis de capacidades organizacionales en investigación y de un análisis del contexto particular enmarcado en la visión bajo las características del isomorfismo competitivo (Meyer, 1979; Fenney, 1980).

Las capacidades organizacionales son concebidas como un constructo en el que se analiza el conjunto interrelacionado de factores, condiciones, limitantes, inventarios y competencias que se despliegan con la finalidad de apoyar los procesos de formulación y gestión efectiva de un mayor número de proyectos y productos de investigación con resultados de generación de valor institucional (Del Río-Cortina, 2020), siendo el contexto de cada institución relevante en el momento de tomar decisiones estratégicas.

La aplicación del análisis de redes sociales en los procesos de investigación de las instituciones de educación superior permite generar la transición del isomorfismo institucional hacia el isomorfismo competitivo en la medida en que le muestra a las instituciones, las facultades, los programas y las escuelas los temas de investigación en los que podrían insertarse de acuerdo con su contexto particular y sus capacidades organizacionales (Poveda, Del Río-Cortina, Diego-Más y Redondo, 2018; Del Río-Cortina, Poveda-Bautista, Redondo-Ortegón y Mejía-Corredor).

El comportamiento de la investigación en el campo administrativo en Colombia ha sido objeto de estudio de varios autores, tal como se plantea en la investigación de Del Río-Cortina (2020). Los autores que han analizado la investigación en el campo administrativo, en su mayor parte, se han enfocado en la evolución de los grupos de investigación nacionales, dejando de lado el análisis comparativo de los procesos de investigación nacionales con respecto a las tendencias internacionales desde las que se desarrolla el conocimiento de vanguardia. 
Malaver (2006) presenta la transición de 32 grupos de investigación en 1997 hacia 2072 en el 2006, mostrando, a su vez, un aumento significativo en los proyectos desarrollados, que pasaron de 845 en el periodo comprendido de 1970-1994, al desarrollo de 4133 en el periodo de 1995-1999, para luego descender a 2739 en el periodo de 2000-2003, con un comportamiento similar en generación de productos de investigación.

En el 2008 en la medición de Colciencias se registran tres grupos del campo administrativo en $\mathrm{A} 1$, y once en A, siendo catorce los grupos considerados élite, con 170 grupos en las otras posiciones (Calderón-Hernández et al., 2010), y continua esta situación de manera similar en mediciones posteriores (Calderón-Hernández et al., 2014).

Es relevante el estudio planteado en el 2017, en el que se evidencia el desarrollo de facultades de administración de «mediano desarrollo de investigación» y de «bajo desarrollo de investigación», de modo que quedan las facultades de administración excluidas de la categoría de «alto desarrollo de investigación» (Calderón-Hernández, Gutiérrez-Vargas y Castaño-Duque, 2017).

Teniendo en cuenta lo anterior, las rutas de fortalecimiento estratégico de la investigación en el campo administrativo deben estar en correspondencia con la visión institucional y la visión de la facultad, el programa o la escuela que se encuentre en la búsqueda de mejoramiento. El fortalecimiento de los procesos de investigación requiere del análisis de los temas de investigación abordados y los objetos de estudio que han sido determinados como referentes dentro de un proceso de isomorfismo competitivo mediado por los costos de transacción. Este proceso de mediación supone tener a los mejores investigadores para desarrollar las temáticas seleccionadas como idóneas, siendo un esfuerzo analítico que se enfoca en la proyección institucional considerando las capacidades organizacionales pertinentes (Del Río-Cortina, 2020). 
En cuanto a la aplicación del análisis de redes, desde los primeros trabajos de Barnes (1954) y Bott (1957) se intentó describir las estructuras sociales y relacionales en términos de redes en el interior de la sociometría norteamericana, encontrándose las redes supeditadas a los conjuntos relacionales, sociales o interpersonales, al crear uniones de individuos, organizaciones o flujos de información desde la perspectiva de grupos. Estos, para el presente caso, se encuentran conformados por los nodos institucionales, los cuales son facultades, programas de administración y escuelas de negocios que representan los objetos de estudio y los nodos temáticos, como, por ejemplo, los temas más importantes de las ciencias administrativas abordados por dichos objetos de estudio.

Desde esta misma perspectiva y de acuerdo con los planteamientos de Watts (2004), es posible encontrar diferentes tipos de relaciones directas entre nodos, lo que genera pequeños mundos de interacciones, con lo cual resultan subestructuras que pueden evolucionar, de mínimamente conectadas hacia una conexión total.

Con el fin de determinar los temas relevantes en ciencias administrativas se plantearon los objetivos específicos de identificar las instituciones representativas en el campo administrativo por regiones; visualizar la relación entre las instituciones representativas y los temas abordados y analizar la relación entre instituciones representativas y temas abordados.

Para dar cumplimiento a los objetivos de esta investigación en los aspectos metodológicos, se abordó la identificación de metodologías. En este sentido, se acogen los conceptos de análisis de redes sociales (Borgatti, Everett y Freeman, 2002), de modo que presentan las principales instituciones por región dentro de un análisis muestral. En lo que corresponde al análisis de redes aplicado a temas de investigación relevantes, se desarrolló una descripción técnica de la manera en la que se aplica la teoría de redes.

En los resultados se identificaron las mejores facultades, programas de administración y escuelas de negocios en el ámbito mundial, evaluando las posiciones del ranquin de Shanghái 
de 2018 (Academic Ranking of World Universities [ARWU], 2018), y en el ámbito nacional, de acuerdo con los resultados de los mejores grupos de investigación en ciencias administrativas registrados en Colciencias (2017).

Se determinó una muestra de instituciones nacionales e internacionales y se identificaron los temas abordados por las diferentes instituciones de la muestra utilizando análisis de contenido (Barringer, Jones y Neubaum, 2005), con la anterior información, se aplicó análisis de redes (Borgatti et al., 2002), lo cual genera el grafo dirigido de visualización de las interacciones de los objetos de estudio con respecto a los temas. También, se diferenciaron los objetos de estudio por regiones, se visualizaron las relaciones entre objetos de estudio y temas abordados, tomando la medida de centralidad de acuerdo con la tipología del nodo y utilizando las medidas de authorities y hubs, del algoritmo hits (Bihari y Kumar, 2015), con lo cual se determinó la importancia de los nodos en la red.

Los resultados permitieron la identificación de nodos temáticos de alta recurrencia, en conjunto con nodos temáticos especializados; se generó la identificación de las variaciones de las posiciones en el ranquin de los objetos de estudio internacional al año 2020 y de los grupos de investigación asociados con los objetos de estudio colombianos considerando la medición del 2018; esto, complementando la evaluación inicial. Finalmente, se abordó la discusión de los resultados y se generaron las conclusiones correspondientes.

\section{Materiales y métodos}

Este es un estudio de tipo analítico (Hernández, Fernández y Baptista, 2013) en el que se generó un modelo relacional de las interacciones entre las instituciones y las temáticas en el campo administrativo aplicando teoría de redes (Masuda y Lambiotte, 2020; Mesa-Angulo et al., 2020).

Con el fin de identificar las instituciones más relevantes en el ámbito mundial, se seleccionó el organismo acreditador más importante debido a su mayor representatividad, en razón a su enfoque investigativo, su rigurosidad y tradición, a saber la Association to Advance 
Collegiate Schools of Business (AACSB): (Guillotin y Mangematin, 2015); a su vez, se seleccionó el ranquin mundial más prominente en cuanto a ciencias administrativas, considerando su rigurosidad y la disponibilidad de datos, el ranquin de Shanghái de 2018 (ARWU, 2018), y como un proceso complementario se analizaron las posiciones de las instituciones académicas para el 2020.

Basados en datos del 2018, inicialmente se identificaron 140 objetos de estudio en el ámbito mundial, ubicados en ocho regiones que se encuentran tanto en el organismo acreditador AACSB como en el ranquin de Shanghái (Tabla 1).

Tabla 1. Distribución de nodos institucionales en el ámbito mundial

\begin{tabular}{|c|c|c|}
\hline Región & $\begin{array}{c}\text { Número de nodos } \\
\text { institucionales }\end{array}$ & $\begin{array}{c}\text { Porcentaje de } \\
\text { representatividad por } \\
\text { región }\end{array}$ \\
\hline África & 1 & $0,71 \%$ \\
\hline Asia & 16 & $11,42 \%$ \\
\hline Centroamérica & 1 & $0,71 \%$ \\
\hline Europa & 24 & $17,14 \%$ \\
\hline Norteamérica & 83 & $59,28 \%$ \\
\hline Oceanía & 10 & $7,14 \%$ \\
\hline Oriente Medio & 1 & $0,71 \%$ \\
\hline Suramérica & 4 & $2,85 \%$ \\
\hline Total & 140 & $99,96 \%$ \\
\hline
\end{tabular}

Fuente. Elaboración propia.

Se determinó una muestra estratificada por regiones del $30 \%$, con un número de 42 objetos de estudio, los cuales fueron seleccionados de acuerdo con la representatividad porcentual por cada región (Tabla 2). La representatividad porcentual se calculó considerando el número de objetos de estudio por región con respecto al total. 
Tabla 2. Distribución de la muestra de nodos institucionales en el ámbito mundial

\begin{tabular}{|c|c|c|}
\hline Región & Muestra & $\begin{array}{c}\text { Porcentaje de } \\
\text { representatividad por } \\
\text { región }\end{array}$ \\
\hline África & 1 & $0,71 \%$ \\
\hline Asia & 4 & $2,85 \%$ \\
\hline Centroamérica & 1 & $0,71 \%$ \\
\hline Europa & 5 & $3,57 \%$ \\
\hline Norteamérica & 24 & $17,14 \%$ \\
\hline Oceanía & 2 & $1,42 \%$ \\
\hline Oriente Medio & 1 & $0,71 \%$ \\
\hline Sur América & 4 & $2,85 \%$ \\
\hline Total & 42 & $29,96 \%$ \\
\hline
\end{tabular}

Fuente. Elaboración propia.

Lo anterior fue complementado con facultades, programas de administración y escuelas de negocios de excelencia en el ámbito colombiano, en un ejercicio de identificación de nodos institucionales nacionales que se basó en el análisis de los mejores grupos de investigación en ciencias administrativas, de acuerdo con los datos de Colciencias (2017). Se obtuvo un listado de diez nodos, de los cuales tres hacen parte del listado anterior de nodos institucionales mundiales: la Facultad de Ciencias Administrativas y de Negocios del ICESI, la Escuela de Gestión de la Universidad de los Andes y la Escuela de Gestión de Negocios de la Universidad Nacional de Colombia.

La muestra final se encuentra compuesta por 49 instituciones, distribuidas en África -1—, Asia -4-, Centroamérica -1-, Europa -5-, Norteamérica -24-, Oceanía -2-, Oriente Medio -1-, Sudamérica -4-, y Colombia -10-, con tres nodos que hacen parte tanto de Suramérica como de Colombia.

Después de la identificación de la muestra final se procedió a la aplicación de la técnica de análisis de contenido (Barringer et al., 2005), de modo que se requirió de la revisión de las páginas web de las instituciones con respecto a los temas de investigación abordados, con lo que se obtuvo un listado de 46 temas de investigación denominados «nodos temáticos». 
En el modelo relacional se consideró la visualización de las interacciones entre nodos institucionales y nodos temáticos en el campo administrativo mediante un grafo o red dirigida en la que se aplica análisis de redes (Borgatti et al., 2002). Una red es un formalismo de modelado utilizado para la construcción de esqueletos de sistemas complejos que genera aproximaciones a la valoración de sus propiedades, su emergencia y su autoorganización (Estrada, 2012). En consideración a lo anterior, para esta investigación las redes resultan ser la mejor apuesta metodológica con miras a determinar los temas de investigación relevantes en ciencias administrativas.

Matemáticamente, una red se define como $G=(V, E)$, donde $V$ es el conjunto de nodos o entidades que conforman la red. Para esta investigación, dichas entidades son las instituciones y sus temáticas científicas y académicas. $E$ se conoce como el conjunto de aristas, el enlace o las interacciones entre las entidades $i$ y $j$, considerando que $i \neq j$. Un elemento importante que se debe tener en cuenta para la construcción de una red son las interacciones de este sistema, puesto que se cuenta con limitaciones de información que permitan inferir las relaciones entre nodos de la misma tipología que, en este caso, serían instituciones o temas. Por tal razón, las interacciones solo están definidas entre nodos de diferente naturaleza, a manera de relaciones de utilidad, preferencia o pertinencia, dando lugar a un tipo de redes que se conoce como redes bipartitas (Figura 1).

Figura 1. Red bipartita dirigida

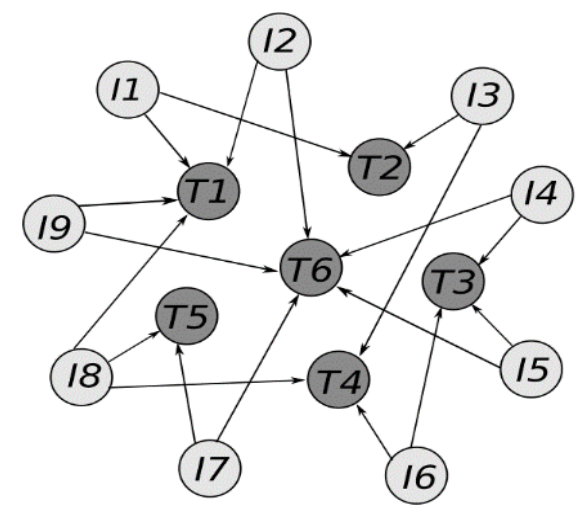

Fuente. Elaboración propia. 
En relación con lo anterior, esta red tiene tres características principales: a) los nodos institucionales corresponden a las mejores facultades, los programas de administración y las escuelas de negocios tanto en el ámbito mundial como en el nacional; mientras b) los nodos temáticos corresponden a los diferentes temas de investigación abordados por los nodos institucionales identificados, y c) la relación entre nodos es dirigida de las instituciones hacia los temas.

Una vez establecidos los nodos, el siguiente paso fue definir la matriz de conectividad o adyacencia de la red. Tal como se planteó en las características de la presente red, se consideró que las relaciones son dirigidas debido a que están basadas en el principio de utilización, con lo que se requiere de una jerarquía en la asociación de los nodos. Con la codificación de la información en estas matrices se estructuraron archivos en formato Edgeslist a fin de realizar la cuantificación de las propiedades y las características del sistema que representa la red en el software Ucinet (Borgatti et al., 2002). En cuanto a las métricas topológicas de la red, en esta investigación se consideraron aquellas que están relacionadas con el rol y la importancia de los nodos en la red, entre las cuales se destacan densidad, grado y centralidad.

La densidad es una medida que permite inferir la homogeneidad o hemofilia de las interacciones entre las entidades que conforman el sistema, es decir, permite valorar la distribución de los temas manejados en cada institución mediante una medida sintética entre cero y uno, siendo uno el mayor valor (Masuda y Lambiotte, 2020; Mesa-Angulo, Gabriel, Ostos-Ortiz y Rentería, 2020).

El grado es una medida utilizada para la valoración de las interacciones de los nodos en una red que se vuelve muy útil cuando se tienen redes poco densas, debido a que permite identificar los nodos más importantes de la red por el rol, la posición y la intermediación de estos en el flujo de la red (Masuda y Lambiotte, 2020; Mesa-Angulo et al., 2020). En este sentido, esta medida permitió identificar tanto las instituciones más prominentes como las temáticas más prestigiosas desde los grados de entrada y salida de la red. 
Por último, para este caso se utilizó la medida de centralidad a fin de determinar los nodos importantes, a partir del cálculo de los hits, un algoritmo que edifica dos valoraciones, los hubs y las authorities (Masuda y Lambiotte, 2020; Mesa-Angulo et al., 2020). Los hubs hacen referencia a los nodos más importantes que generan la información o el flujo de información que para este caso serían las instituciones. Por su parte, las authorities son los temas de investigación más abordados por las instituciones.

En la presente investigación se adoptó la medida de centralidad conforme a la tipología del nodo, aspecto en el que las medidas de las authorities y los hubs del algoritmo de los hits son relevantes (Bihari y Kumar, 2015), así como son consideradas un parámetro que permite determinar la posición de conectividad estratégica de las instituciones y de los nodos temáticos. Esta medida de centralidad indica la influencia de un nodo en una red, debido a que expresa los nodos de la red que poseen un nivel de conexión más alto con respecto a otros nodos bien conectados, teniendo en cuenta la direccionalidad de la relación.

Se generó una revisión de los datos de 2020 en el ranquin de Shanghái en cuanto a los objetos de estudio internacionales y en el proceso de medición de grupos de investigación asociados a los objetos de estudios colombianos; esto, como un proceso complementario de análisis de posibles variaciones en los objetos de estudio.

\section{Resultados}

El proceso de identificación de nodos institucionales muestra 49 nodos representativos de la excelencia en facultades, escuelas y programas de administración tanto mundiales como nacionales (Tabla 3). 
Tabla 3. Nodos institucionales y su ubicación territorial

\section{Nodos institucionales}

Instituto Gordon de ciencias de la Administración

Facultad de Negocios-The Hong Kong Polytechnic University

NUS Business School

Escuela de Negocios HKUST

Escuela de Negocios CUHK

Instituto Centroamericano de Administración de Empresas

Escuela de Negocios Rotterdam

Escuela de Gestión INSEAD

Escuela de Economía y Gestión Tilburg

Facultad de Economía y Negocios Groningen

Escuela de Negocios Aalto

Escuela de Negocios de Harvard

Escuela Wharton

Escuela de gestión Sloan MIT

Universidad de Negocios Smeal

Escuela de Negocios W. P. Carey

Escuela de Gestión Joseph L. Rotman.

Escuela de Gestión Carlson

Universidad de Negocios Scheller

Escuela de Negocios Robert H. Smith

Escuela de Negocios Kelly

Escuela de Negocios Stanford

Escuela de Negocios Columbia

Escuela de Negocios Kenan-Flagler

Universidad de Negocios Cornell

Escuela de Negocios Fuqua

Escuela de Negocios Sauder

Universidad de Negocios J. Mack Robinson

Escuela de Negocios Alberta

Universidad de Negocios Warrington

Escuela de Negocios Red McCombs

Escuela de Negocios Leonard N. Stern

Escuela de Negocios Marshall

Universidad de Negocios Broad

Michigan Ross

Escuela de Negocios UQ

Escuela de Negocios Monash

Escuela de Negocios The Leon Recanati

Escuela de Negocios INSPER

Facultad de Ciencias Administrativas y Económicas ICESI

Escuela de Gestión de la Universidad de los Andes

Programa de Administración-Universidad Nacional de

Colombia

Escuela de Negocios del Rosario

Escuela de Negocios Universidad Eafit

Facultad de Negocios Simón Bolívar

Facultad de Economía y Negocios Universidad Javeriana

Facultad de Economía y Negocios Universidad de Medellín

Facultad de Negocios Universidad Ean

Escuela de Negocios Uninorte

Fuente. Elaboración propia. 
Del análisis de las posibles variaciones de los objetos de estudio internacionales en el periodo 2020 se determinó que, en general, las instituciones por región no presentan una variación significativa en la posición dentro del ranquin de Shanghái (véase la tabla 4). Las instituciones identificadas en el ranquin de Shanghái son aún las más relevantes en las regiones en donde se encuentran.

Tabla 4. Variación en el ranquin de Shanghái de los nodos institucionales al 2020

\begin{tabular}{|c|c|c|c|}
\hline Nodo Institucional & Región & $\begin{array}{l}\text { Ranquin de Shanghái- } \\
\text { administración de } \\
\text { empresas } 2018\end{array}$ & $\begin{array}{l}\text { Ranquin de Shanghái- } \\
\text { administración de } \\
\text { empresas } 2020\end{array}$ \\
\hline Business & \multirow[t]{2}{*}{ África } & $301-400$ & Sale del ranquin \\
\hline Graduate School of Business & & Está fuera el ranquin & $301-400$ \\
\hline HKUST Business School & \multirow[t]{3}{*}{ Asia } & 37 & 31 \\
\hline NUS Business School & & 46 & 49 \\
\hline CUHK Business School, Hong Kong and Shenzhen & & $51-75$ & $51-75$ \\
\hline Erasmus University Rotterdam & \multirow[t]{5}{*}{ Europa } & 5 & 3 \\
\hline $\begin{array}{l}\text { Tilburg School of Economics and Management and } \\
\text { TIAS School for Business and Society }\end{array}$ & & 6 & 6 \\
\hline Groningen Faculty of Economics and Business & & 33 & 38 \\
\hline Aalto School of Business & & 34 & 24 \\
\hline INSEAD Management & & 48 & 16 \\
\hline Columbia Business School & \multirow[t]{15}{*}{ Norteamérica } & 8 & 12 \\
\hline Kelley School of Business & & 11 & 15 \\
\hline Cornell College of Business & & 12 & 31 \\
\hline The Fuqua School of Business & & 13 & 7 \\
\hline Sauder School of Business & & 15 & 26 \\
\hline J. Mack Robinson College of Business & & 16 & 8 \\
\hline Alberta School of Business & & 17 & 34 \\
\hline Joseph L. Rotman School of Management & & 19 & 29 \\
\hline Warrington College of Business Administration & & 21 & 36 \\
\hline Red McCombs School of Business & & 24 & 25 \\
\hline Leonard N. Stern School of Business & & 25 & 29 \\
\hline Kenan-Flagler Business School & & 27 & 19 \\
\hline Marshall School of Business & & 27 & 27 \\
\hline Broad College of Business & & 29 & 22 \\
\hline W. P. Carey School of Business & & 31 & 16 \\
\hline Monash Business School - Monash University & \multirow[t]{2}{*}{ Oceanía } & 22 & $51-75$ \\
\hline UQ Business School & & $51-75$ & $151-200$ \\
\hline $\begin{array}{l}\text { The Leon Recanati Graduate School of Business } \\
\text { Administration }\end{array}$ & Oriente Medio & $101-150$ & $101-150$ \\
\hline
\end{tabular}

Fuente. Elaboración propia. 
En este sentido, en el 2020 se evidencia la salida del ranquin de la institución Gordon Institute of Business Science-University of Pretoria, con respecto a la información presentada en el 2018, ingresando en su reemplazo la institución University of Cape Town como representante de África, con el nodo institucional Graduate Business School.

Además de lo anterior, el análisis de la medición del 2018 en cuanto a las posiciones de los diferentes grupos de investigación asociados a las universidades colombianas evidencia la permanencia de las diferentes instituciones objeto de estudio así: con el grupo Innovar del Caribe de la Universidad del Norte en A1, tanto en el 2017 como en el 2018; el grupo G3Pymes de la Universidad Ean en A1 para el 2017 y el 2018; el Grupo Cultura y Gestión Organizacional de la Universidad de Medellín, que pasa de A hacía A1 del 2017 al 2018; el Grupo Conocimiento, Innovación y Competitividad de la Universidad Javeriana en A, para el 2017 y el 2018; el grupo Gestión Organizacional de la Universidad Simón Bolívar que pasa de A hacia A1 del 2017 al 2018; el Grupo Administración y Organizaciones de la Universidad EAFIT en A1 para el 2017 y el 2018; el grupo de Investigación en Dirección y Gerencia de la Universidad del Rosario en A1 para el 2017 y el 2018; el grupo Cultura Organizacional y Gestión Humana categorizado en A1 para el 2017 y el 2018; el grupo Historia y Empresariado de la Universidad de los Andes en A1 para el 2017 y el 2018; y, finalmente, el grupo Inversión, Financiación y Control de la Universidad ICESI, categorizado en A1 para el 2017 y el 2018.

La Facultad de Ciencias Administrativas y de Negocios de la universidad ICESI, la Escuela de Gestión de la Universidad de los Andes y la Escuela de Gestión de Negocios de la Universidad Nacional de Colombia son referentes en el ámbito colombiano al encontrarse tanto en la identificación mundial como nacional.

Norteamérica es el territorio que concentra el mayor porcentaje de nodos institucionales con un $49 \%$, representado por un número de veinticuatro nodos, de los cuales veintiuno corresponden a Estados Unidos, con el 43 \% de los nodos institucionales, de modo que es referente mundial en términos de temas de investigación en ciencias administrativas con nodos altamente representativos como, por ejemplo, la Escuela de Negocios de Columbia, la 
Universidad de Negocios Cornell, la Escuela de Gestión Sloan del MIT, la Universidad de Negocios J. Mack Robinson, la Escuela de Negocios W. P. Carey, la Escuela de Negocios KenanFlagler, la Universidad de Negocios Broad y la Escuela de Negocios de la Universidad de Harvard.

En el momento de visualizar los nodos institucionales de Estados Unidos y aplicar el parámetro de centralidad de Eigenvector (Bihari y Kumar, 2015), se presenta un grafo en el que sobresalen los nodos de la Escuela de Negocios de Columbia, la Escuela de Gestión Sloan del MIT, la Universidad de Negocios Cornell y la Universidad de Negocios J. Mack Robinson, mientras que, al mismo tiempo, se evidencia el aislamiento de tres temas que no son abordados, los cuales son comportamiento organizacional, gerencia de proyectos y turismo (Figura 2).

Figura 2. Nodos institucionales ubicados en Estados Unidos

Turismo

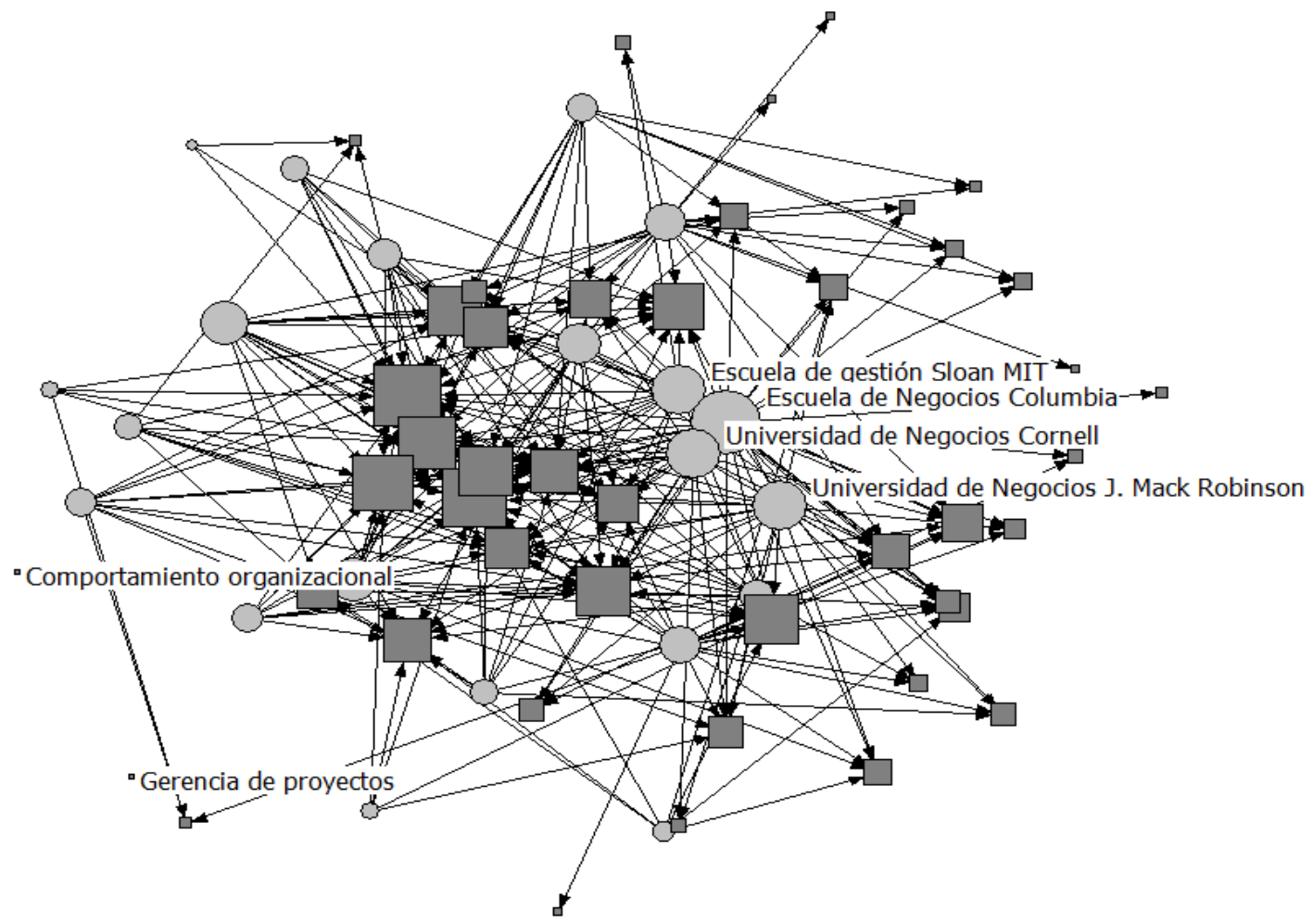

Fuente. Elaboración propia mediante el software Ucinet. 
En este punto, Estados Unidos maneja un sistema de ciencia, tecnología e innovación que perfila sus esfuerzos en el conocimiento aplicado en busca del mejoramiento del desempeño de su sistema productivo, con lo cual se contempla la incidencia de las investigaciones en las empresas con un enfoque de generación de empleos, crecimiento y competitividad que involucra en las propuestas de investigación una serie de stakeholders tales como las instituciones de educación superior, las instituciones de soporte, el sistema de ciencias y las organizaciones empresariales con sus redes, entre otras organizaciones de investigación y desarrollo, con participación activa de clústeres de las industrias farmacéutica, biotecnológica y de tecnología de la información, bajo el liderazgo del sector productivo, combinando procesos de fricción empresarial con la generación de valor compartido (Porter y Kramer, 2011).

Siguiendo con los resultados, se identifican 46 temas o nodos temáticos, de los cuales se destacan como temas de alta recurrencia el comportamiento financiero, el emprendimiento, la globalización y el liderazgo, mientras se identifican como nodos especializados el género, las neurociencias, la gerencia de proyectos, la comercialización minorista, el liderazgo de producción pública y el turismo (Tabla 5).

Tabla 5. Nodos temáticos y recurrencia

\begin{tabular}{|l|c|}
\hline \multicolumn{1}{|c|}{ Nodos temáticos } & $\begin{array}{c}\text { Número de nodos institucionales } \\
\text { conectados al nodo temático }\end{array}$ \\
\hline Comportamiento financiero & 34 nodos conectados \\
\hline Emprendimiento & 33 nodos conectados \\
\hline Globalización & 30 nodos conectados \\
\hline Liderazgo & 30 nodos conectados \\
\hline Innovación & 27 nodos conectados \\
\hline Negocios y ambiente & 26 nodos conectados \\
\hline Operaciones y gestión de suministros & 23 nodos conectados \\
\hline Mercadeo & 23 nodos conectados \\
\hline Gerencia y estudios internacionales & 22 nodos conectados \\
\hline Transporte y logística & 22 nodos conectados \\
\hline Contabilidad y finanzas & 21 nodos conectados \\
\hline Crecimiento e innovación & 19 nodos conectados \\
\hline Gestión de servicio y operaciones & 18 nodos conectados \\
\hline Análisis de negocios & 18 nodos conectados \\
\hline Gestión de riesgos y decisiones & 18 nodos conectados \\
\hline Digitalización & 17 nodos conectados \\
\hline Estrategia y competitividad & 15 nodos conectados \\
\hline Economía & 15 nodos conectados \\
\hline
\end{tabular}




\begin{tabular}{|l|c|}
\hline Salud & 14 nodos conectados \\
\hline Empresas sociales & 12 nodos conectados \\
\hline Análisis del consumidor & 11 nodos conectados \\
\hline Mercado laboral & 11 nodos conectados \\
\hline Estudios de gestión avanzados & 10 nodos conectados \\
\hline Corporaciones y sociedad & 10 nodos conectados \\
\hline Talento humano & 10 nodos conectados \\
\hline Evaluación financiera & 8 nodos conectados \\
\hline Ética de negocios & 6 nodos conectados \\
\hline Gobierno corporativo & 6 nodos conectados \\
\hline Firmas familiares globales & 6 nodos conectados \\
\hline Análisis de personas & 6 nodos conectados \\
\hline Pequeños negocios & 6 nodos conectados \\
\hline Mercados emergentes & 5 nodos conectados \\
\hline Gestión de hospitalidad & 5 nodos conectados \\
\hline Comportamiento organizacional & 5 nodos conectados \\
\hline Método de casos & 4 nodos conectados \\
\hline Competitividad & 4 nodos conectados \\
\hline Capital privado & 4 nodos conectados \\
\hline Bienes raíces & 4 nodos conectados \\
\hline Historia empresarial & 3 nodos conectados \\
\hline Negocio de los deportes & 3 nodos conectados \\
\hline Género & 2 nodos conectados \\
\hline Neurociencias & 2 nodos conectados \\
\hline Gerencia de proyectos & 2 nodos conectados \\
\hline Comercialización minorista & 2 nodos conectados \\
\hline Liderazgo de educación pública & 1 nodo conectado \\
\hline Turismo & 1 nodo conectado \\
\hline & \\
\hline
\end{tabular}

Fuente. Elaboración propia.

Al aplicar la medida de centralidad de grado nodal (Borgatti et al., 2002), en cuanto la determinante del tamaño de los nodos, se genera un grafo en el que se evidencian diferencias marcadas de conexión que muestran los nodos temáticos de finanzas, emprendimiento, globalización y liderazgo como nodos centrales y de mayor tamaño debido a su alta recurrencia; mientras que se presentan los nodos de género, neurociencias, gerencia de proyectos, comercialización minorista, liderazgo de educación pública y turismo como nodos periféricos de tamaño mínimo, ya que son temas especializados (Figura 3). 
Figura 3. Medida de centralidad de grado nodal

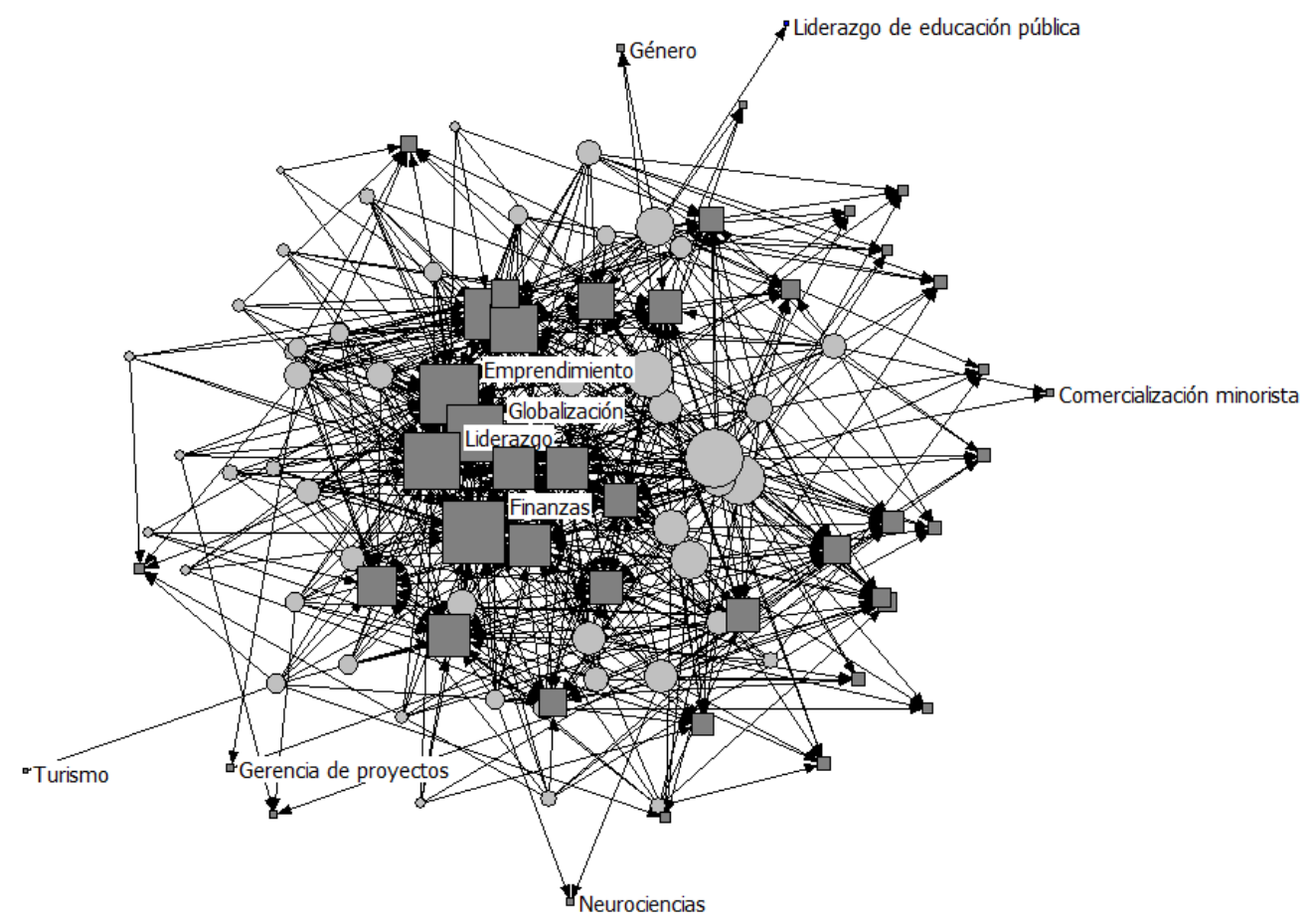

Fuente. Elaboración propia, mediante el software Ucinet.

Al aplicar la medida de centralidad de Eigenvector (Bihari y Kumar, 2015), la cual evidencia los nodos de la red de mayor conexión en referencia con otros nodos bien conectados, se identifican, en orden de conectividad, los nodos temáticos de finanzas con 0,193, emprendimiento con 0,188 , globalización con 0,183 , liderazgo con 0,176 , innovación con 0,158 , y negocios y ambiente con 0,154 (Figura 4). 
Figura 4. Nodos temáticos de alta recurrencia

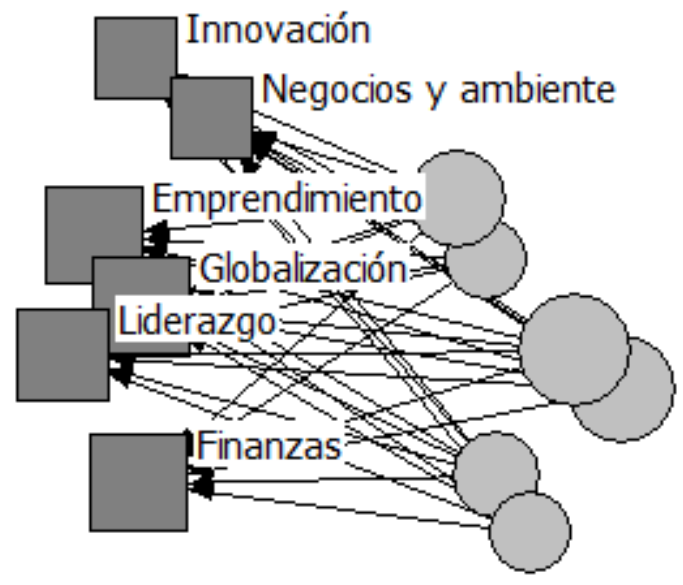

Fuente. Elaboración propia mediante el software Ucinet.

Por otra parte, al orientar el grafo hacia los nodos de menor conexión con respecto a los nodos mayor conectados se identifican los nodos temáticos especializados (Figura 5).

Figura 5. Nodos temáticos especializados

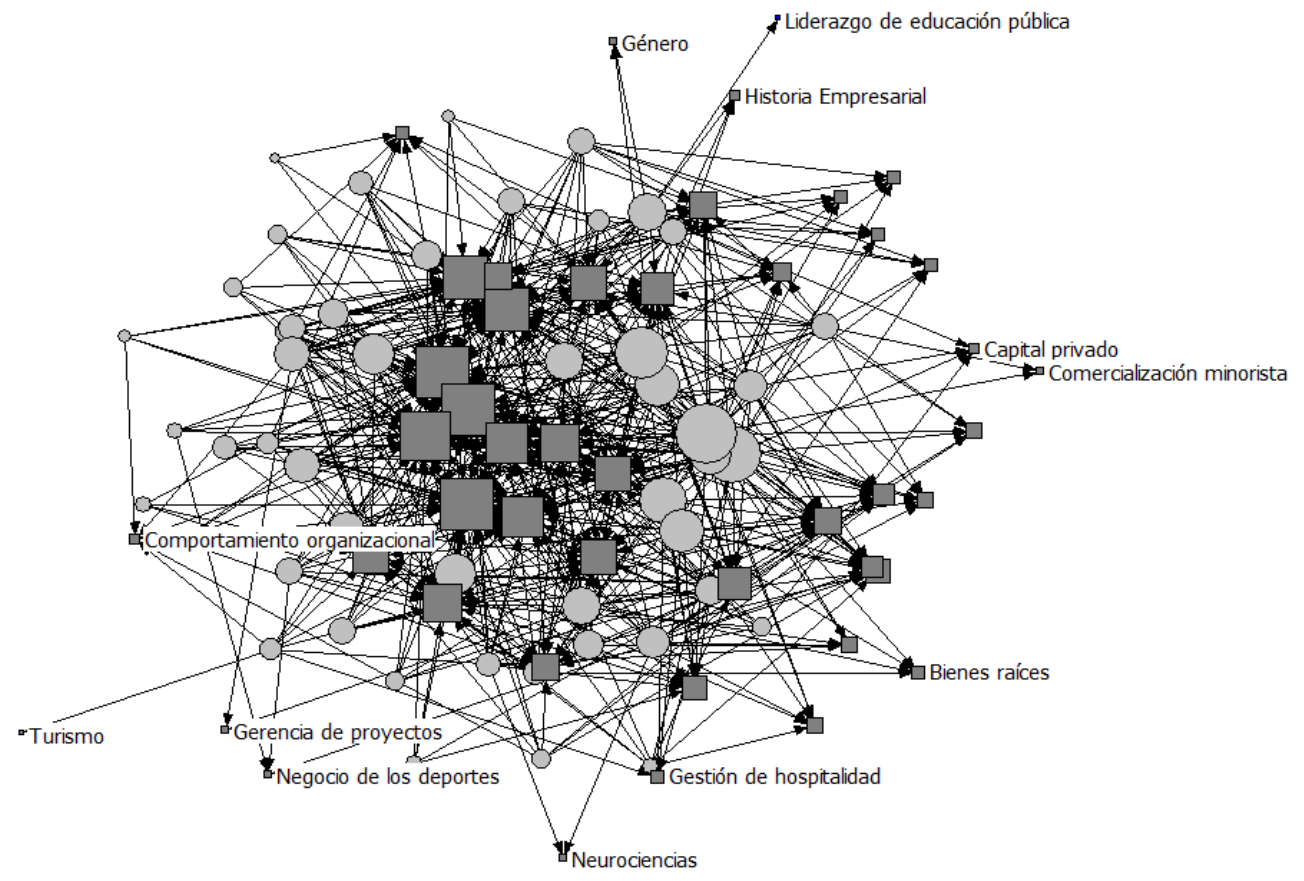

Fuente. Elaboración propia, mediante el software Ucinet. 
Con lo anterior se identifican los nodos especializados de turismo con 0,003 , liderazgo de educación pública con 0,007 , neurociencias con 0,010 , negocio de deportes y gerencia de proyectos con 0,013 , género con 0,015 , comercialización minorista con 0,016 , historia empresarial con 0,022, comportamiento organizacional con 0,024, capital privado con 0,027, bienes raíces con 0,031 y gestión de hospitalidad con 0,032.

\section{Discusión}

En un mundo globalizado con problemáticas complejas se requiere de aproximaciones a la realidad mediante herramientas que faciliten la toma de decisiones e incidan en el mejoramiento del sistema productivo y de la sociedad en general.

En este punto, en el que se presentan nuevos desafíos para las instituciones académicas que se encuentran contemplados en las funciones sustantivas de docencia, investigación y proyección social, desarrollándose al interior de una perspectiva global (Guillotin y Mangematin, 2015), se requiere del contacto con el sistema productivo para dinamizar la innovación y apropiación social del conocimiento en el interior de un proceso de mejoramiento.

Un proceso de mejora continua institucional que debe estar en concordancia con los lineamientos de Colciencias (2017) y del Consejo Nacional de Acreditación (CNA, 2013), como organismos que marcan la pauta del accionar de las instituciones de educación superior en Colombia, en términos de investigación y estructura curricular, respectivamente; además, teniendo en cuenta los lineamientos del Ministerio de Educación Nacional (2014), debe identificar las mejores prácticas institucionales dentro de un análisis de benchmarking acorde a las características de cada institución (Nazarko et al., 2009).

Desarrollar este tipo de investigaciones en las instituciones de educación superior implica una serie de cuestionamientos relacionados con la manera de identificar los objetos de estudio que se analizarán, de modo que se presenta literatura limitada en cuanto a la forma de seleccionar dichos objetos de estudio con algunos casos representativos en Australia 
(Stella y Woodhouse, 2007), Canadá (Proulx, 2007), Estados Unidos (Achemeier y Simpson, 2005), China y Taiwán Mok y Chan, 2008), y Europa (European Centre for Strategic Management of Universities [ESMU], 2008).

En este sentido, la iniciativa de benchmarking europea muestra que la sistematización del proceso es fundamental en el propósito de obtener resultados que realmente reflejen el estado de los objetos de estudio analizados, generando un escenario que permita replicar las buenas prácticas (ESMU, 2008).

Los diferentes casos de aplicación mencionados carecen de información suficiente en cuanto a la forma en la que se identificaron los objetos de estudio, de modo que surge el análisis de redes como una metodología que permite generar una descripción y estudio de las estructuras que se forman a partir de la interacción de diferentes organizaciones o participantes del ejercicio académico en la medida en que desarrollan actividades conjuntas, se comunican, coinciden y colaboran, a través de diversos procesos o acuerdos que pueden gestarse de manera bilateral o multilateral. De este modo, emerge una estructura basada en la existencia de una red social que en esta investigación se encuentra representada por la estructura de visualización de las relaciones entre nodos institucionales y nodos temáticos, de manera que permite una mejor identificación de objetos de estudio.

De acuerdo con Nazarko et al. (2009), el benchmarking es la mejor forma de desarrollar el mejoramiento continuo en las instituciones de educación superior, dado que es un proceso en el que es de vital importancia la identificación de los nodos según el contexto. Además, al presentarse información limitada en cuanto a una apropiada identificación de nodos, la adopción de metodologías validadas se hace necesaria, de modo que se propone en el presente documento el análisis de redes como una metodología idónea para la visualización de nodos institucionales y sus relaciones con nodos temáticos con el fin de desarrollar un posterior benchmarking de facultades, escuelas y programas en ciencias administrativas. 


\section{Conclusiones}

El desarrollo de proyectos de investigación desde una perspectiva integral, acorde a la planeación estratégica, debe contribuir al desarrollo tanto de la comunidad académica —con sus diferentes grupos de interés- como del sector productivo, de instancias gubernamentales y de la comunidad en general, propiciando la generación y el incremento de capacidades institucionales. Así mismo, el análisis de los temas de investigación relevantes en ciencias administrativas es un ejercicio de benchmarking que permite enfocar los esfuerzos en cuanto a la visualización de nodos institucionales representativos, desde la perspectiva de facultades, escuelas o programas de administración que abordan temas considerados estratégicos en los niveles global y regional, ya que son temas acordes con el pensamiento estratégico de la institución que desarrolla el análisis y que, a su vez, se corresponden con sus capacidades organizacionales.

A partir de las estructuras de interconexión, representadas por los grafos, es posible analizar la relevancia de los nodos temáticos con respecto a los nodos institucionales, identificando temáticas abordadas por un número significativo de facultades, escuelas y programas de administración y, a su vez, identificando temáticas especializadas consideradas por un número muy limitado de nodos institucionales.

Aplicando análisis de redes es posible construir grafos que muestran la manera en la que se dan las relaciones de adopción de temáticas por parte de los nodos institucionales en el interior de una red en la que confluyen temáticas comunes a gran número de nodos institucionales, en conjunto con temáticas especializadas asumidas por muy pocos nodos.

En la medida en que los hallazgos muestran solo tres nodos institucionales de excelencia que son referente para Colombia, al encontrarse en el ranquin de Shanghái en el interior de la identificación de nodos nacionales, se evidencia una oportunidad de mejora para los diferentes nodos institucionales nacionales, de modo que quedan ampliamente rezagados con respecto a Estados Unidos, territorio en donde se presenta la mayor participación de 
nodos institucionales en el interior del ranquin de Shanghái y referente mundial de los temas de investigación en ciencias administrativas.

A fin de comprender mejor este rezago en la producción científica en ciencias administrativas se hace necesario evaluar la filosofía y el desempeño del Sistema de Ciencia, Tecnología e Investigación y, a su vez, determinar las condiciones de funcionamiento del ecosistema de innovación.

De esta forma, la identificación de temas de alta recurrencia y de temas especializados es insuficiente para determinar los ejes temáticos a abordar si no se tiene claridad en la visión de la facultad, la escuela o el programa, con lo cual la presente investigación se convierte en un insumo para la toma de decisiones que debe ser complementado con el conocimiento interno en términos de capacidades organizacionales y la apropiación del pensamiento estratégico con énfasis en la visión institucional. Además, con el conocimiento del contexto requieren una aproximación al Sistema de Ciencia, Tecnología e Innovación y al funcionamiento del ecosistema de innovación colombianos.

Los hallazgos muestran que se presenta un escenario en el que los nodos institucionales se enfrentan a la decisión de abordar temas comunes a la mayoría o concentrarse en propuestas altamente diferenciadoras y muy particulares, con lo cual los nodos institucionales deberían enfocarse en desarrollar parámetros de isomorfismo competitivo abordando temáticas estratégicas.

Finalmente, la selección de temas de investigación en ciencias administrativas requiere del análisis del contexto en el que se encuentra cada facultad, escuela o programa, con el fin de desarrollar investigaciones acordes a las necesidades de las comunidades académicas y empresariales en las que los diferentes nodos institucionales ejercen influencia directa, aquellos en los que la complejidad de los proyectos de investigación en el campo administrativo implica la toma de decisiones institucionales entre el isomorfismo institucional y el isomorfismo competitivo; esto con el fin de implementar estrategias acordes al direccionamiento estratégico. 


\section{Referencias}

Academic Ranking of World Universities. (2018). Ranking académico de las Universidades del Mundo del 2018. Shanghairanking.com. Recuperado de https://bit.ly/33CcMzr

Achemeier, S. D.; Simpson, R.D. (2005). Practical considerations when using benchmarking for accountability in higher education. Innovative Higher Education, 30, 117-128. DOI: https://doi.org/10.1007/s10755-005-5014-3

Association to Advance Collegiate Schools of Business. (2020). Guiding principles and standards for business accreditation: engagement, innovation, impact. AACSB. Recuperado de https://bit.ly/325A7sa

Association to Advance Collegiate Schools of Business. (2012). Pact of research, a guide for business schools. AACSB.

Barnes, J. A. (1954). Class and committee in a Norwegian island parish. Human Relations, 7(1), 39-58. DOI: https://doi.org/10.1177/001872675400700102

Barringer, B. R.; Jones, F. F.; Neubaum, D. O. (2005). A quantitative content analysis of the characteristics of rapid-growth firms and their founders. Journal of Business Venturing, 20(5), 663-687. DOI: https://doi.org/10.1016/i.jbusvent.2004.03.004

Bihari, A.; Kumar, M. (2015). Key author analysis in research professionals' relationship network using citation indices and centrality. Procedia Computer Science, 57, 606-613. DOI: https://doi.org/10.1016/j.procs.2015.07.414

Borgatti, S.; Everett, M.; Freeman, L. (2002). Netdraw network visualization. Boston: Analytic Technologies.

Bott, E. (1957). Family and social network. Londres: Tavistock Publications.

Calderón-Hernández, G.; Arrubla-Zapata, J.; Castaño-Duque, G.; Gutiérrez-Vargas, L.; Posada-Bernal, R.; Ruiz-Berrío, A.; Serna-Gómez, H.; Vivares-Vergara, J. (2010). La investigación en administración en Colombia. Condiciones para la generación de conocimiento, investigadores, institucionalización y producción científica. Medellín: Asociación Colombiana de Facultades de Administración - Capítulo Antioquia.

Calderón-Hernández, G.; Castaño-Duque, G.; Lozada-Barahona, N.; Gutiérrez-Vargas, L.; PérezHerrera, P.; Posada-Bernal, R.; García-Serna, L.; Castro-Escobar, R. (2014). Generación de conocimiento en los grupos élite de investigación en administración en Colombia. Bogotá: Universidad Nacional de Colombia.

Calderón-Hernández, G.; Gutiérrez-Vargas, L.; Castaño-Duque, G. (2017). La investigación en las facultades de administración de Colombia. Revista Lasallista de investigación, 14(1), 42-55. DOI: https://doi.org/10.22507/rli.v14n1a3

Colciencias. (2017). Guía para el reconocimiento y medición de grupos de investigación e investigadores. Bogotá: Colciencias. 
Consejo Nacional de Acreditación (2013). Lineamientos para la acreditación de programas de pregrado. Bogotá: CNA.

Dalkir, K. (2005). Knowledge management in theory and practice. Boston: Elsevier.

Danielson, L.; Doolittle, J.; Bradley, R. (2007). Professional development, capacity building, and research needs: critical issues for response to intervention implementation. School Psychology Review, 36(4), 632-637. DOI: https://doi.org/10.1080/02796015.2007.12087922

David, F. R. (2013). Conceptos de administración estratégica. Ciudad de México: Pearson Education.

Del Río-Cortina, A. (2020). Incidencia de una estructura de gestión estratégica de proyectos de investigación en el incremento de capacidades organizacionales en facultades de administración (Tesis doctoral). Universidad Ean. Bogotá, Colombia. Recuperado de https://bit.ly/3N1RhsI

Del Río-Cortina, A.; Poveda-Bautista, R.; Redondo-Ortegón, J.; Mejía-Corredor, C. (2019). Percepción de importancia de las variables de aprobación para proyectos de investigación, caso de estudio Fundación Universitaria Cafam. Gestión Ingenio y Sociedad, 3(2), 1-7. Recuperado de https://bit.ly/3HFyOkz

Dimaggio, P. J.; Powel, W. W. (1983). The iron cage revisited: institutional isomorphism and collective rationality in organizational fields. American Sociological Review, 48(2), 147-160. DOI: https://doi.org/10.2307/2095101

Estrada, E. (2012). The structure of complex networks: theory and applications. Nueva York: Oxford University Press. DOI: https://doi.org/10.1093/acprof:oso/9780199591756.001.0001

European Centre for Strategic Management of Universities. (2008). Benchmarking in European Higher Education. Recuperado de https://bit.ly/3DYiEza

Fenney, M. L. (1980). The effects of environmental characteristics on the structure of hospital clusters. Administrative Science Quarterly, 25(3), 484-510. DOI: https://doi.org/10.2307/2392265

Guillotin, B.; Mangematin, V. (2015). Internationalization strategies of business schools: how flat is the world? Thunderbird International Business Review, 57(5), 343-357. DOI: https://doi.org/10.1002/tie.21705

Hernández, R.; Fernández, C.; Baptista, P. (2013). Metodología de la investigación. Ciudad de México: McGraw-Hill.

Malaver, F. (2006). El despegue de la investigación colombiana en administración: análisis de sus avances en el período 2000-2006. Cuadernos de Administración, 19(32), 71-109. Recuperado de https://bit.ly/30mZEQF

Masuda, N.; Lambiotte, R. (2020). Guide to temporal networks. Singapur: World Scientific. DOI: https://doi.org/10.1142/q0268 
Mesa-Angulo, O. P.; Gabriel, F. J.; Ostos-Ortiz, O. L.; Rentería, R. R. (2020). Modelo de vigilancia tecnológica e inteligencia estratégica: evaluación de nuevos programas académicos de la Universidad Santo Tomás. Bogotá: Ediciones USTA. DOI: https://doi.org/10.15332/li.lib.2020.00255

Meyer, J. W. (1979). The impact of the centralization of educational funding and control on state and local organizational governance. Stanford: Institute for Research on Educational Finance and Governance - Stanford University.

Mihaela, D.; Amalia, D.; Bogdan, G. (2015). The partnership between academic and business environment. Procedia-Social and Behavioral Sciences, 180, 298-304. DOI: https://doi.org/10.1016/i.sbspro.2015.02.120

Ministerio de Educación Nacional. (2014). Reflexiones para la política de internacionalización de la educación superior en Colombia. Bogotá: MEN - OCyT.

Mok, H. J.; Chan, Y. (2008). International benchmarking with the best universities: policy and practice in Mainland China and Tajwan. Higher Education Policy, 21, 469-486. DOI: https://doi.org/10.1057/hep.2008.21

Nazarko, J.; Kuzmic, K.; Szubzda-Prutis, E.; Urban, J., (2009). The general concept of benchmarking and its application in higher education in Europe. Higher Education in Europe, 34(3-4), $497-$ 510. DOI: https://doi.org/10.1080/03797720903356677

Porter, M. E.; Kramer, M. R. (2011). La creación de valor compartido. Harvard Business Review. Recuperado de https://bit.ly/3IRImZQ

Poveda, R.; Del Río-Cortina, A.; Diego-Más, J. A.; Redondo, J. M. (2018). Definición de un índice para la selección de proyectos de investigación basado en el proceso analítico de red (ANP). Ponencia presentada en el 22nd International Congress on Project Management and Engineering. Madrid, España, 11-13 de julio.

Proulx, R. (2007). Higher education ranking and league tables: lessons learned from benchmarking. Higher Education in Europe. 32(1), 71-82. DOI: https://doi.org/10.1080/03797720701618898

Salcedo-Serna, M.; Londoño-Cardozo, J.; Hernández-Madroñero, I. (2017). Tendencias de formación posgradual y áreas de investigación en Administración de Empresas. Estudio comparativo en América. Logos, Ciencia \& Tecnología, 9(2), 134-152. DOI: https://doi.org/10.22335/rlct.v9i2.442

Stella, A.; Woodhouse, D. (2007). Benchmarking in Australian Higher Education: A Thematic Analysis of AUQA Audit Reports. AUQA Occasional Publications. 13, 5-59.

Watts, D. J. (2004). Small worlds: The dynamics of networks between order and randomness. Princeton University Press. 